University of South Carolina

Scholar Commons

2-12-2013

\title{
Neutrino Beam Constraints on Flavor-Diagonal Lorentz Violation
}

Brett David Altschul

altschul@mailbox.sc.edu

Follow this and additional works at: https://scholarcommons.sc.edu/phys_facpub

Part of the Physics Commons

\section{Publication Info}

Physical Review D, Volume 87, Issue 9, 2013.

(C) 2013 American Physical Society

This Article is brought to you by the Physics and Astronomy, Department of at Scholar Commons. It has been accepted for inclusion in Faculty Publications by an authorized administrator of Scholar Commons. For more information, please contact digres@mailbox.sc.edu. 
arXiv:1302.2598

\title{
Neutrino Beam Constraints on Flavor-Diagonal Lorentz Violation
}

\author{
Brett Altschul] \\ Department of Physics and Astronomy \\ University of South Carolina \\ Columbia, SC 29208 USA
}

\begin{abstract}
Breaking of isotropy and Lorentz boost invariance in the dynamics of second-generation leptons would lead to direction-dependent changes in the lifetimes of charged pions. This would make the intensity of a neutrino beam produced via pion decay a function of the beam orientation. The experimental signature of this phenomenonsidereal variations in the event rate at a downstream neutrino detector - has already been studied, in searches for Lorentz-violating neutrino oscillations. Existing analyses of MINOS near detector data can be used to constrain the flavor-diagonal Lorentz violation coefficients affecting muon neutrino speeds at roughly the $10^{-5}$ level.
\end{abstract}

\footnotetext{
${ }^{1}$ baltschu@physics.sc.edu
} 


\section{Introduction}

One exotic topic in elementary particle physics that has received a significant amount of attention of late is the possibility that the fundamental laws of nature may not be perfectly invariant under Lorentz and CPT symmetries [1]. Thus far, there is no compelling experimental reason to doubt that these symmetries are exact. However, there are important theoretical reasons to be interested in the topic. Many prospective theories of quantum gravity may involve the breaking of these symmetries, at least in certain regimes. The mechanisms for the symmetry breaking include spontaneous breaking in string theory [2, 3] and theories of vector fields [4], effects in loop quantum gravity [5, 6] and non-commutative geometry [7, 8], Lorentz violation through spacetime-varying couplings [9, 10], and anomalous breaking of Lorentz and CPT symmetries [11].

The discovery of any kind of Lorentz or CPT violation would be extraordinarily important - a sure sign of new physics, with a fundamentally different structure from anything that has so far been observed. Such a discovery would provide a remarkable window onto a fundamentally new and different physical regime. This fact makes the subject area quite interesting, for both theoretical and experimental research programs. Most recent theoretical work is now performed within the context of effective quantum field theory. There is an extensively-studied effective field theory known as the standard model extension (SME) that contains all possible translation-invariant but Lorentz-violating operators that may be constructed out of known standard model fields.

In the SME, the effects of Lorentz-violating operators are parameterized by small tensor-valued coefficients [12, 13. In scenarios in which the Lorentz violation arises spontaneously, these tensor coefficients represent the nonzero vacuum expectation values of tensor-valued fields. Because CPT violation in a quantum field theory that is both stable and unitary automatically entails the existence of Lorentz violation as well [14], the SME is automatically the general effective field theory for describing CPT-violating physics.

As a systematic effective field theory, the SME contains, in principle, an infinite number of possible Lorentz-violating operators. However, all but a small number of these operators are expected to be suppressed at modest experimental energies. For low-energy experiments, the most relevant subset of the general theory is the minimal SME, which involves only those forms of Lorentz and CPT violation that are gauge invariant, local, and superficially renormalizable. This minimal theory has become the standard framework used for parameterizing experimental Lorentz tests. Recent searches for Lorentz violation have included studies of matter-antimatter asymmetries for trapped charged particles [15, 16, 17] and bound state systems [18, 19], measurements of muon properties [20, 21], analyses of the behavior of spin-polarized matter [22], frequency standard comparisons [23, 24, 25, 26], Michelson-Morley experiments with cryogenic resonators [27, 28, 29, 30, 31], Doppler effect measurements [32, 33], measurements of neutral meson oscillations [34, 35, 36, 37, 38, 39], polarization measurements on the light from cosmological sources [40, 41, 42, 43], high-energy astrophysical tests [44, 45, 46, 47, 48], 
precision tests of gravity [49, 50], and others. The results of these experiments set constraints on the various SME coefficients, and up-to-date information about most of these constraints may be found in [51].

The neutrino sector of the standard model is one of the most difficult to study, since neutrinos only interact weakly. However, there have been a number of analyses of how Lorentz violation could affect neutrino oscillation phenomena. The general framework for studying SME-driven neutrino oscillations was given in [52]. Based on this work, there have been experimental analyses of the results from the MINOS [53, 54, 55, 56], LSND [57], IceCube [58], Double Chooz [59], and MiniBooNE [60] experiments. This paper will look at a different kind of neutrino Lorentz violation, one not involving oscillations; however, the ultimate physical observable is the same as in some of the oscillation analyses. This means that elements drawn from previous data analyses can immediately be carried over into the present work.

Measuring flavor-diagonal Lorentz violation for neutrinos can be a challenge. The MINOS analyses were intended as a search for off-diagonal SME coefficients - for example, looking for oscillations $\nu_{\mu} \rightarrow \nu_{x}$ along a baseline whose direction is changing slowly with the Earth's rotation. Particle oscillation experiments can be extremely sensitive to small effects, because they measure the quantum mechanically coherent build-up of tiny effects. In contrast, the conventional way to identify neutrino Lorentz violation that does not involve flavor-changing operators is to look for deviations from the expected neutrino velocity $v_{\nu}=1$ [61, 62, 63, 64]. Measuring the velocities of neutral particles such as neutrinos is much more difficult than equivalent measurements with charged species; for charged leptons, it is possible to measure their velocities indirectly, through their interactions with photons, because it is the four-velocity that governs how a charge interacts with the electromagnetic field.

This paper will continue to focus on those forms of Lorentz violation that can modify the neutrino velocities most pronouncedly. However, rather than direct measurements of $v_{\nu}$, the focus will be on other effects generated by the same SME coefficients. In particular, changes to the limiting neutrino velocity will also affect the rate at which neutrinos are produced in weak decays. Most of the constraints described in this paper concern coefficients affecting $\nu_{\mu}$ propagation that have never previously been bounded. Despite a similarity in the notation (when flavor indices are suppressed), these are not the same coefficients studied in [53, 55]; those results concerned coefficients that were off-diagonal in flavor space and so could be constrained by looking at flavor oscillation phenomena.

This paper is organized as follows. Section 2 will discuss how Lorentz violation in the lepton sector affects the decay rates for charged pions. Section 3 will discuss the influence of the specific experimental geometry of the NuMI beam on pion decay observables. New bounds based on previous analyses of the MINOS data are presented in section 4, followed by a discussion of the outlook for future experimental improvements. 


\section{Lorentz Violation in Pion Decay}

Most of the SME coefficients for the first-generation fermions that make up everyday matter have been measured quite precisely. However, forms of Lorentz violation involving only second-generation particles are quite a bit more difficult to constrain. Experiments that are sensitive to second-generation Lorentz violation must involve either unstable charged particles or weakly coupled neutrinos. Nonetheless, there are observables that depend rather sensitively on the SME coefficients for the second-generation species. In particular, there are flavor-diagonal effects that could produce the same kind of signature in the MINOS near detector as Lorentz-violating oscillations between $\nu_{\mu}$ and another species $\nu_{x}$. These phenomena cause the initial intensity of the neutrino beam to depend on the direction in which the beam is pointing. Periodic changes in the neutrino beam intensity would then change the number of muon charged current events recorded in the near detector, giving the same experimental signature as Lorentz-violating neutrino oscillations. However, comparison measurements using both the near and far detectors will eliminate the dependence on the initial intensity of the neutrino beam; they can therefore be used to distinguish changes to the neutrino speeds from Lorentz-violating oscillation effects.

The most prominent reason that the NuMI beam intensity might vary with the sidereal orientation is that Lorentz violation for the second-generation leptons affects the lifetimes of the pions that decay to produce the beam. In any decay, the dispersion relation for the daughter particles has a strong effect on the decay rate, since it determines the amount of phase space available for the decay products. Modifications of the muon and neutrino energy-momentum relations will therefore change the rate at which pions decay to produce those particles. There are also changes, of comparable size, to the weak interaction matrix element for the process.

The details of the decay rate have been worked out [65], to leading order in the relevant Lorentz violation coefficients. The SME Lagrange density relevant for the leptons in this scenario is

$$
\mathcal{L}=i \bar{L}\left(\gamma^{\mu}+c_{L}^{\nu \mu} \gamma_{\nu}\right) D_{\mu} L+i \bar{R}\left(\gamma^{\mu}+c_{R}^{\nu \mu} \gamma_{\nu}\right) D_{\mu} R+\text { mass terms }
$$

where $L$ and $R$ are the left- and right-chiral lepton multiplets,

$$
L=\left[\begin{array}{c}
\nu_{L} \\
\ell_{L}
\end{array}\right], \quad R=\left[\ell_{R}\right]
$$

and $D_{\mu}$ is the covariant derivative, containing the electromagnetic and weak gauge interactions. There are separate coefficients for the left-chiral and right-chiral leptons; these can be alternately expressed in terms of Lorentz violation coefficients $c^{\nu \mu}$ and $d^{\nu \mu}$, where

$c_{L}^{\nu \mu}=c^{\nu \mu}+d^{\nu \mu}$ and $c_{R}^{\nu \mu}=c^{\nu \mu}-d^{\nu \mu}$. However, since only left-chiral particles undergo charged current weak interactions, only $c_{L}$ affects the decay rate of charged pions. The 
effects of flavor-diagonal minimal SME coefficients other than the $c$ and $d$ are suppressed at relativistic energies.

Moreover, it is important to note that $S U(2)_{L}$ gauge invariance requires that the Lorentz violation coefficients $\left(c_{L}\right)^{\nu \mu}$ be the same for the left-chiral charged lepton and its corresponding neutrino. This means that it is not possible to have neutrinos with Lorentzviolating dispersion relations without having the same kinds of modifications also applying to the charged leptons - unless the chiral gauge invariance (which is responsible for such features as lepton universality) is abandoned.

The $c_{L}$ coefficients together form a traceless background tensor. The isotropic element of this tensor, $\left(c_{L}\right)_{00}$, endows spacetime with a preferred frame structure. This leads to an isotropic violation of Lorentz boost symmetry. The other eight independent coefficients also break spatial isotropy as well as boost invariance. Although Lorentz violation is often accompanied by CPT violation, the effects of the $c_{L}$ coefficients are all CPT-even.

The pion decay rate $\Gamma$ depends, at leading order, on the isotropic coefficient $\left(c_{L}\right)_{00}$, evaluated in the rest frame of the decaying pion. The dependence is 65 ]

$$
\Gamma=\Gamma_{0}\left[1+\frac{4}{1-m_{\mu}^{2} / m_{\pi}^{2}}\left(c_{L}\right)_{00}\right],
$$

where $\Gamma_{0}$ is the rest frame decay rate in the absence of Lorentz violation. The changes that lead to the modified $\Gamma_{0}$ are of two types. The first changes are in the invariant matrix element for the process. A gauge field couples to the average velocity of the incoming and outgoing particles at an interaction vertex; this kind of velocity coupling is most familiar for electromagnetic interactions (in the form of the classical Lorentz force law), but the weak coupling behaves similarly. Having modified particle speeds therefore affects the pion decay matrix element. Changes of the second type are kinematic in nature. Having unusual dispersion relations for the outgoing particles changes the phase space available for the decay, which in turn changes $\Gamma$. The phase space contributions are particularly important in this case; they lead to the effects of $\left(c_{L}\right)_{00}$ being enhanced by the similarity in the pion and muon masses. Using the physical values of these quantities, the decay rate is $\Gamma=\Gamma_{0}\left[1+9.4\left(c_{L}\right)_{00}\right]$.

In the pion rest frame, the total decay rate depends only on the $\left(c_{L}\right)_{00}$ in that frame. However, because decaying pions are typically moving relativistically relative to the laboratory, $\left(c_{L}\right)_{00}$ depends on the direction of that motion. This gives the pion lifetime observed in the laboratory a sidereal dependence, as the pion beam direction moves with the rotation of the Earth. Notably, the sidereal dependence of the decay rate (and thus the neutrino beam strength) is equally sensitive to violations of isotropy and violations of boost invariance. This is a sharp contrast with the sensitivities seen in many low-energy laboratory experiments, which are typically much more sensitive to anisotropy than boost violation. The reason for the parity in sensitivity in this case is that the decaying pions are moving relativistically in the laboratory, and this motion creates a strong sensitivity to relativistic effects; as the Earthbound laboratory rotates, this provides a way of testing 
whether the behavior of the pions is the same when they are highly boosted in different directions.

Experimental constraints on the SME coefficients are conventionally expressed using a set of Sun-centered celestial equatorial coordinates [66]. The origin of these coordinates lies at the center of the Sun. The $Z$-axis points parallel to the Earth's rotation axis; the $X$-axis points toward the vernal equinox point on the celestial sphere; and the $Y$ axis is determined by the right hand rule. Time in these coordinates is $T$; however, it is generally advantageous to choose a local time coordinate $T_{\oplus}$ so that at $T_{\oplus}=0$, the laboratory $y$-direction coincides with the $Y$-direction in the Sun-centered frame.

The isotropic $\left(c_{L}\right)_{00}$ in the pion rest frame is

$$
\left(c_{L}\right)_{00}=\gamma_{\pi}^{2}\left[\left(c_{L}\right)_{T T}+\left(c_{L}\right)_{(T J)}\left(v_{\pi}\right)_{J}+\left(c_{L}\right)_{J K}\left(v_{\pi}\right)_{J}\left(v_{\pi}\right)_{K}\right],
$$

in terms of the pion velocity $\vec{v}_{\pi}$, its Lorentz factor $\gamma_{\pi}$, and the SME coefficients in the Suncentered frame. The capital letter indices indicate that the implied sums over the spatial coordinate indices $J$ and $K$ are being performed in the Sun-centered frame. The velocities associated with the movement of the Earthbound laboratory are all nonrelativistic, so $\vec{v}_{\pi}$ is essentially the the pion's velocity relative to the lab.

When the particles involved in the decay are moving ultrarelativistically in the lab frame, the relevant $\left(c_{L}\right)_{00}$ effectively depends on the speed $v_{\pi} \approx 1$ only through the Lorentz factor $\gamma_{\pi}$. Then the linear combination $\left(c_{L}\right)_{T T}+\left(c_{L}\right)_{(T J)}\left(\hat{v}_{\pi}\right)_{J}+\left(c_{L}\right)_{J K}\left(\hat{v}_{\pi}\right)_{J}\left(\hat{v}_{\pi}\right)_{K}$, which depends only on the direction $\hat{v}_{\pi}$ of the overall motion, becomes the key quantity that determines the energy-momentum relation for ultrarelativistic leptons. The dispersion relation for the highly relativistic decay products (as observed in the lab frame) is $E=[1-$ $\left.\left(c_{L}\right)_{T T}-\left(c_{L}\right)_{(T J)}\left(\hat{v}_{\pi}\right)_{J}-\left(c_{L}\right)_{J K}\left(\hat{v}_{\pi}\right)_{J}\left(\hat{v}_{\pi}\right)_{K}\right]|\vec{p}|$. This takes into account relativistic beaming, which causes the decay products to be emitted along essentially the same direction as the parent pion.

The dependence of $\Gamma$ on the pion beam direction is not the only manifestation of anisotropy. In the pion rest frame, the decay is actually anisotropic. Decay products are more likely to emerge in some directions than others. In the pion frame, the rate for the decay channel with daughter particles emerging in directions $\hat{v}_{\mu}$ and $\hat{v}_{\nu}=-\hat{v}_{\mu}$ may contain terms proportional to $\left(c_{L}\right)_{(T J)}\left(\hat{v}_{\nu}\right)_{J}$ or $\left(c_{L}\right)_{J K}\left(\hat{v}_{\nu}\right)_{J}\left(\hat{v}_{\nu}\right)_{K}$ at leading order. However, in the total decay rate, which is found by integrating over all directions, all the anisotropic terms cancel; the increased rates for decays into certain directions compensate for decreased rates in other directions, leaving the total decay rate only dependent on the lone isotropic term $\left(c_{L}\right)_{00}$. Moreover, the anisotropy of the decay products is difficult to measure, because relativistic effects ensure that the decay products are beamed into a narrow pencil of angles around the direction $\hat{v}_{\pi}$ of the pion's motion. It is worth noting that beaming is also responsible for the fact that it is only the dispersion relation for particles moving essentially in the $\hat{v}_{\pi}$-direction that determines the laboratory frame decay rate. That this should be the case can be seen from the fact that if the decay rate were (cumbersomely) evaluated in the lab frame, the phase space available for the decay products would only 
depend on their energy-momentum relations within their narrow range of allowed angles around $\hat{v}_{\pi}$.

While a full understanding of the decay anisotropy in the pion rest frame would be fairly interesting, it is beyond the scope of the present analysis, since this information is not required in order to place order of magnitude bounds on the $c_{L}$ parameters that are flavor diagonal (meaning those $c_{L}$ coefficients associated with operators that do not mix multiple neutrino flavors). In fact, calculating the angular dependence of the decay would require a substantially more elaborate generalization of the calculation in [65]. There are no approximations apparent that could reliably be used to simplify such a calculation. Phase space estimates are sometimes useful in evaluations of the rates for Lorentz-violating processes. These estimates involve ignoring the effects of Lorentz violation on the dynamical matrix elements involved and studying merely how the Lorentz violation coefficients affect the phase space kinematics. This strategy is typically useful when looking at processes that are normally forbidden by Lorentz symmetry - that is, those for which the phase space available for the process is conventionally zero [44, 67, 68]. It is not surprising that in such situations, the changes to the kinematics should predominate. However, the calculations in [65] show that for pion decay, the effects of the $c_{L}$ coefficients on the dynamics and kinematics are comparable in size; a phase space estimate of the pion decay rate will not be quantitatively accurate.

Another simplification would apply if both the daughter particles were moving ultrarelativistically in the center-of-mass frame, as is the case for the $\pi^{+} \rightarrow e^{+}+\nu_{e}$ decay mode. In such a scenario, there is no dynamical or kinematical distinction between the two outgoing particles in the absence of Lorentz violation [because of the Lorentz-invariant theory's $S U(2)_{L}$ and CP symmetries]. The equivalence of the daughter particles, which travel in antipodal directions in the center-of-mass frame, would preclude any anisotropy that had different rates of neutrino production in the $\hat{p}$ - and $-\hat{p}$-directions. This would rule out all odd multipole patterns; in particular, there could be no dependence on the dipolar $\left(c_{L}\right)_{(0 j)}$ coefficients, and any anisotropy would have to come from the $\left(c_{L}\right)_{j k}$. Unfortunately, for the decay to a muon-neutrino pair, the muon is not energetic enough for this simplification to occur.

\section{$3 \quad$ Effects of Experimental Geometry}

In a neutrino oscillation experiment like MINOS, a detector intercepts the neutrino beam near the beam's point of origin. If Lorentz violation in the neutrino sector leads to sidereal oscillations in the strength of the neutrino beam, the oscillations will be detectable using the near detector. In this section, we will focus specifically on understanding how to make such a measurement using the NuMI neutrino beam and the MINOS detector located at Fermilab, because there is more information available about possible sidereal oscillations for MINOS than for other high-energy neutrino experiments. 
The most elementary constraint based on the variability of the pion decay rate arises from the fact that there can be a threshold boost beyond which the decay $\pi^{+} \rightarrow \mu^{+}+\nu_{\mu}$ becomes energetically impossible. This effect is nonperturbative in the $c_{L}$ coefficients, and so it was not included in the perturbative analysis that led to (3). Nonetheless, if $E_{\pi}>\sqrt{\left(m_{\pi}^{2}-m_{\mu}^{2}\right) / 2\left[-\left(c_{L}\right)_{T T}-\left(c_{L}\right)_{(T J)}\left(v_{\pi}\right)_{J}-\left(c_{L}\right)_{J K}\left(v_{\pi}\right)_{J}\left(v_{\pi}\right)_{K}\right]}$ (when the square root is real), the greater-than-normal growth of the muon and neutrino energies as functions of $|\vec{p}|$ means that there is not enough energy to produce daughter particles with the required momentum [69, 70]. The primary pion decay channel becomes disallowed. (If the square root is imaginary, no such threshold exists; this occurs if the neutrino velocity in the relevant direction is less than the speed of light, and so the neutrino energy $E_{\nu}=v_{\nu} p_{\nu}$ grows more slowly than in the Lorentz-invariant theory.)

Naturally, the existence of an upper threshold for the pion decay process could lead to sidereal variations in the beam. The nonoccurrence of the decay above a certain pion energy will affect the neutrino intensity. As the beam direction rotates with the Earth, the threshold will vary, and a variable fraction of the expected population of high energy neutrinos will fail to be produced. However, a constraint derived from an analysis of this phenomenon appears to be less sensitive than one that can be extracted from an analysis of the decay rate based on the perturbative formula (3).

The energy distribution of the neutrinos in the NuMI beam is somewhat complicated. However, in order to get an order of magnitude constraint on the $c_{L}$ coefficients for second-generation leptons, we shall treat the problem as the decay of a $\sim 6 \mathrm{GeV}$ pion, without regard to the direction of the decay neutrino. This energy is chosen to simulate a neutrino beam with a peak at $\sim 3 \mathrm{GeV}$. A full analysis of the beam energy structure would introduce a number of additional complications. The degree of forward beaming depends on the progenitor pion energy, and this interacts nontrivially with the effects of Lorentz violation as well. While the total decay rate $\Gamma$ depends only on the isotropic $\left(c_{L}\right)_{00}$ in the pion rest frame, the amplitudes for specific angular decay channels depend on the anisotropic coefficients contracted with the momentum vectors of the daughter particles. Certain neutrino production directions will be favored. Especially at lower pion energies, where beaming is less pronounced, this can affect the energy composition of the NuMI beam. In contrast, at high energies, the decay neutrinos are strongly aligned into the observable beam, and the sensitivity to Lorentz violation is simultaneously higher, because of a larger factor $\gamma_{\pi}$ appearing in the boosted expression for $\left(c_{L}\right)_{00}$.

Other experimental complications may be dealt with more explicitly. The result (3), in conjunction with the formula (44) for $\left(c_{L}\right)_{00}$, gives the instantaneous decay rate for pions moving in a particular direction. However, what is measured with an observation of the neutrino beam strength is not the decay rate but the total number of pions that manage to decay during the neutrino beam production process. This means that the length of the pion decay pipe affects the magnitude of any variations in the neutrino beam strength. If the pion decay rate, as measured in the laboratory frame, is $\Gamma / \gamma_{\pi}$, then for pions moving at speed $v_{\pi} \approx 1$ along a decay pipe of length $D$, the fraction that decay over the length of 
the pipe is $P(D)=1-e^{-\Gamma D / \gamma_{\pi}}$. The fractional difference in $P(D)$, if there is a fractional change in the decay rate $\Delta \Gamma / \Gamma_{0}$, is

$$
\left(\left.\frac{1}{P} \frac{d P}{d \Gamma}\right|_{\Gamma=\Gamma_{0}}\right) \Delta \Gamma=\left(\frac{\Delta \Gamma}{\Gamma_{0}}\right) \frac{\Gamma_{0} D / \gamma_{\pi}}{e^{\Gamma_{0} D / \gamma_{\pi}}-1},
$$

with a factor of $\Gamma_{0} / \Gamma_{0}$ inserted to make the expression of product of two dimensionless quantities. Note that for $D \gg \gamma_{\pi} \Gamma_{0}^{-1}$, essentially all the pions have time to decay, so a change in the decay rate does not affect the total beam strength. In contrast, if $D \ll$ $\gamma_{\pi} \Gamma_{0}^{-1}$, the decaying fraction is $P(D) \approx \Gamma D / \gamma_{\pi}$, and the fractional change in the number of decays is simply $\Delta \Gamma / \Gamma_{0}$. As $D$ increases, the sensitivity of the beam intensity is suppressed; however, there is also naturally an improvement in statistics at the detector, as the total number of the neutrinos in the beam grows. The optimal sensitivity for a monoenergetic beam will occur in the vicinity of $D \sim \gamma_{\pi} \Gamma_{0}^{-1}$. For the NuMI beam, the decay pipe length is $677 \mathrm{~m}$ long, chosen to be comparable to $\gamma_{\pi} \Gamma_{0}^{-1}=\gamma_{\pi}\left(2.6 \times 10^{-8} \mathrm{~s}\right)$ for pions with $\mathrm{GeV}$ energies. For a $6.0-\mathrm{GeV}$ pion, the suppression factor $\Gamma_{0} D / \gamma_{\pi}\left(e^{\Gamma_{0} D / \gamma_{\pi}}-1\right)$ is 0.31 .

Finally, the most important influence that the specific geometry of the MINOS experiment has on the possibility of sidereal oscillations in the detector event rate comes from the details of how the pion decay direction changes with time. Let the detector be located at colatitude $\chi\left(\chi=42.18^{\circ}\right.$ for the MINOS near detector at Fermilab), and let $(\theta, \phi)$ be spherical coordinates describing the angle between the beam direction and the local zenith direction $\left(\theta=93.27^{\circ}\right)$ and the azimuthal angle in the plane of the Earth's surface, measured starting eastward from south $\left(\phi=203.91^{\circ}\right)$. Then the beam direction at a local time $T_{\oplus}=0$ is [71]

$$
\begin{aligned}
\hat{v}_{\pi}= & N_{1} \hat{X}+N_{2} \hat{Y}+N_{3} \hat{Z} \\
= & (\cos \chi \sin \theta \cos \phi+\sin \chi \cos \theta) \hat{X}+(\sin \theta \sin \phi) \hat{Y} \\
& +(-\sin \chi \sin \theta \cos \phi+\cos \chi \cos \theta) \hat{Z} \\
= & -0.715 \hat{X}-0.405 \hat{Y}+0.571 \hat{Z} .
\end{aligned}
$$

As the Earth rotates with sidereal frequency $\omega_{\oplus}$, the key quantity $\left(c_{L}\right)_{00}$ varies according to

$$
\left(c_{L}\right)_{00}=\gamma_{\pi}^{2}\left[\mathcal{A}_{0}+\mathcal{A}_{\omega} \cos \left(\omega_{\oplus} T_{\oplus}\right)+\mathcal{B}_{\omega} \sin \left(\omega_{\oplus} T_{\oplus}\right)+\mathcal{A}_{2 \omega} \cos \left(2 \omega_{\oplus} T_{\oplus}\right)+\mathcal{B}_{2 \omega} \sin \left(2 \omega_{\oplus} T_{\oplus}\right)\right]
$$

where the coefficients are

$$
\begin{aligned}
\mathcal{A}_{0} & =\left(c_{L}\right)_{T T}+N_{3}\left(c_{L}\right)_{(T Z)}+N_{3}^{2}\left(c_{L}\right)_{Z Z}+\frac{1}{2}\left(1-N_{3}^{2}\right)\left[\left(c_{L}\right)_{X X}+\left(c_{L}\right)_{Y Y}\right] \\
\mathcal{A}_{\omega} & =N_{1}\left(c_{L}\right)_{(T X)}+N_{1} N_{3}\left(c_{L}\right)_{(X Z)}+N_{2}\left(c_{L}\right)_{(T Y)}+N_{2} N_{3}\left(c_{L}\right)_{(Y Z)} \\
\mathcal{B}_{\omega} & =-N_{2}\left(c_{L}\right)_{(T X)}-N_{2} N_{3}\left(c_{L}\right)_{(X Z)}+N_{1}\left(c_{L}\right)_{(T Y)}+N_{1} N_{3}\left(c_{L}\right)_{(Y Z)}
\end{aligned}
$$




$$
\begin{aligned}
\mathcal{A}_{2 \omega} & =\frac{1}{2}\left(N_{1}^{2}-N_{2}^{2}\right)\left(c_{L}\right)_{-}+N_{1} N_{2}\left(c_{L}\right)_{(X Y)} \\
\mathcal{B}_{2 \omega} & =-N_{1} N_{2}\left(c_{L}\right)_{-}+\frac{1}{2}\left(N_{1}^{2}-N_{2}^{2}\right)\left(c_{L}\right)_{(X Y)},
\end{aligned}
$$

with $\left(c_{L}\right)_{-}=\left(c_{L}\right)_{X X}-\left(c_{L}\right)_{Y Y}$.

The ultimate experimental observable - the number of muon events recorded in the detector - will therefore be modulated at the frequencies $\omega_{\oplus}$ and $2 \omega_{\oplus}$. Collecting all the factors to determine the sensitivity, the fractional modulation in the normalized event rate will have amplitudes equal to the $\mathcal{A}$ and $\mathcal{B}$ coefficients times a sensitivity factor $\mathcal{S}$. The sensitivity factor is assembled from several pieces. There is the boost factor $\gamma_{\pi}^{2}$ [from (4) and (9), which represents the enhancement of the boost invariance violation effects controlled by $\left(c_{L}\right)_{00}$ due to the relativistic motion of the center of mass. There is the also $c_{L}$-dependent term from $\Gamma / \Gamma_{0}$, which tells how much $\left(c_{L}\right)_{00}$ affects the decay rate; and the result (5), which describes how the modified decay rate $\Gamma$ in turn affects the beam intensity. These combine to give

$$
\mathcal{S}=\left(\gamma_{\pi}^{2}\right)\left(\frac{4}{1-m_{\mu}^{2} / m_{\pi}^{2}}\right)\left(\frac{\Gamma_{0} D / \gamma_{\pi}}{e^{\Gamma_{0} D / \gamma_{\pi}}-1}\right)=5.4 \times 10^{3} .
$$

The largest part of the enhancement obviously derives from the highly relativistic nature of the progenitor pions.

\section{Conclusions From MINOS Data}

In order to constrain the $c_{L}$ coefficients for the second-generation leptons using MINOS data, an analysis of the observed beam intensity versus sidereal time is required. Fortunately, the core of the necessary analysis has already been performed by the experimenters; this was the basis of the papers [53, 55], the conservative spirit of whose analyses will be followed here. The observables for the scenario with a direction-dependent rate of oscillations $\nu_{\mu} \rightarrow \nu_{x}$ and the scenario with a similarly direction-dependent pion decay rate are the same: a change in the number of muon charged current events that indicate interactions of the beam neutrinos with the detector. Since [53, 55] found no significant evidence for sidereal oscillations in the number of muon neutrinos in the beam, there can be no evidence of a variation in the charged pion decay rate in these data sets either. Instead, there will be constraints on the anisotropic $c_{L}$ parameters.

The MINOS analyses found no evidence of oscillations that reached the $3 \sigma$ level of significance. In fact, the observed signal levels were well below such a level. The analysis in [53] determined the Fast Fourier Transform power present in each quadrature mode at frequencies $\omega_{\oplus}$ and $2 \omega_{\oplus}$, as well as additional higher harmonics. The level of statistical noise was characteristic of a $1 \sigma$ dispersion in these power values of $1.8 \times 10^{-2}$, when the data were normalized to the total event rate (which was set by the rate of protons on 


\begin{tabular}{|c|c|}
\hline Coefficient & Bound \\
\hline$\left|\left(c_{L}\right)_{(T X)}\right|$ & $2.3 \times 10^{-5}$ \\
$\left|\left(c_{L}\right)_{(T Y)}\right|$ & $2.3 \times 10^{-5}$ \\
$\left|\left(c_{L}\right)_{-}\right|$ & $5.4 \times 10^{-5}$ \\
$\left|\left(c_{L}\right)_{(X Y)}\right|$ & $5.4 \times 10^{-5}$ \\
$\left|\left(c_{L}\right)_{(X Z)}\right|$ & $4.0 \times 10^{-5}$ \\
$\left|\left(c_{L}\right)_{(Y Z)}\right|$ & $4.0 \times 10^{-5}$ \\
\hline
\end{tabular}

Table 1: Bounds on the magnitudes of the individual $c_{L}$ coefficients for second-generation leptons, assuming only one coefficient at a time is nonvanishing.

target in the collisions that originate the pions). Thus a $3 \sigma$ bound on the normalized amplitudes $\mathcal{S} \mathcal{A}$ and $\mathcal{S B}$ of the oscillations at the two lowest frequencies is $5.4 \times 10^{-2}$. This corresponds to constraints on the separate $\mathcal{A}$ and $\mathcal{B}$ coefficients of approximately $\times 10^{-5}$.

The results of [55] are similar but used an antineutrino data set with slightly less statistical power. Note that even in the presence of nonzero $d$ coefficients, the antineutrino data provide complementary constraints on the same $c_{L}$ coefficients, which govern the dispersion relations for left-handed neutrinos and their right-handed antiparticles.

Of the nine Lorentz-violating components of $\left(c_{L}\right)^{\nu \mu}$, six can be constrained by looking at sidereal variations in the event rates at multiple neutrino experiments. The ones that cannot be constrained this way include $\left(c_{L}\right)_{T T}$, which is purely isotropic, as well as $\left(c_{L}\right)_{(T Z)}$ and $\left(c_{L}\right)_{Z Z}$, which describe the effects of a preferred direction lying parallel to the Earth's axis. In the spherical harmonic notation of [72], the $c_{L}$ coefficients affecting only a single generation of leptons are equivalent to the $\left(c_{\mathrm{of}}^{4}\right)_{j m}$, with highest weights up to $j=2$; the six subject to constraint according to this method are those with $m \neq 0$ in celestial equatorial coordinates. For a single particular experiment, four linear combinations of the remaining six parameters may be constrained. These linear combinations are represented by the $\mathcal{A}$ and $\mathcal{B}$ coefficients.

If the absence of sidereal oscillations in the signal is not due to a coincidental cancellation among the coefficients, it is possible to set order of magnitude constraints on the six separate $\left|\left(c_{L}\right)_{(T X)}\right|,\left|\left(c_{L}\right)_{(T Y)}\right|,\left|\left(c_{L}\right)_{-}\right|,\left|\left(c_{L}\right)_{(X Y)}\right|,\left|\left(c_{L}\right)_{(X Z)}\right|$ and $\left|\left(c_{L}\right)_{(Y Z)}\right|$ coefficients. This may be done by setting all but one of the coefficients to zero, then finding the value of the remaining coefficient that is required to raise one of the $\mathcal{A}$ or $\mathcal{B}$ to an observable $3 \sigma$ level. The results are given in table 1.

These order of magnitude constraints on the anisotropy coefficients, at the $10^{-5}$ level, are comparable to bounds on the time-averaged neutrino-light velocity difference $v_{\nu}-1$ based on terrestrial experiments with muon neutrinos. The sidereally averaged neutrino speed measured in such experiments depends on the six constrained here and also on the remaining coefficients $\left(c_{L}\right)_{T T},\left(c_{L}\right)_{(T Z)}$, and $\left(c_{L}\right)_{Z Z}$, as outlined in [65]. Each such speed 
measurement depends on a particular linear combination of the $c_{L}$ coefficients involved.

In future searches for anisotropic neutrino oscillations, based on muon neutrino disappearance, it should be possible to add additional sensitivity to the flavor-diagonal coefficients discussed here. The sensitivity to the $c_{L}$ coefficients that affect solely secondgeneration leptons derives from the way the form of Lorentz violation involved affect the decay of the pion progenitors of the beam neutrinos. The sensitivities of future measurements obviously depend most on the energies of the pions involved; greater $\gamma_{\pi}$ values will generally improve constraints. The position and orientation of the beam have lesser influences, as does the length of the pion decay region. A very long decay pipe actually wipes out sensitivity in the lower-energy component of the neutrino beam; the beam intensity is independent of $c_{L}$ at a given energy if essentially all the pions in the relevant energy range have sufficient time to decay. It is possible that this kind of shift toward higher energies may actually be advantageous; although the signal is lost at lower energies, the sensitivity to the $c_{L}$ coefficients increases with $\gamma_{\pi}^{2}$, and the quality of the relativistic beaming approximation also increases with energy. A full understanding of these effects will require more detailed understanding of the parent pion and daughter neutrino beams' energy structures.

The current level of sensitivity is much lower than the sensitivity to SME parameters that induce oscillations. It is also poorer than the $\sim 10^{-11}$ sensitivity that has been obtained from the observed absence of the photon decay reaction $\gamma \rightarrow \mu^{-}+\mu^{+}$for cosmic ray photons [73]; however, because the photon is a spin-1 state, the daughter particles in such a reaction would necessarily have parallel spins, which makes such constraints only sensitive to the spin-averaged $c$ coefficients, rather than the left-chiral $c_{L}$. On the other hand, the sensitivity of the new method discussed in this paper is comparable to that achieved for flavor-diagonal effects using direct time-of-flight measurements, and this new strategy can represent an important tool for constraining boost and isotropy violation in the difficult-to-access neutrino sector.

\section{References}

[1] For overviews of recent work on Lorentz violation, see D. Mattingly, Living Rev. Rel. 8, 5 (2005) and the contents of Proceedings of the Fifth Meeting on CPT and Lorentz Symmetry, edited by V. A. Kostelecký (World Scientific, Singapore, 2011).

[2] V. A. Kostelecký, S. Samuel, Phys. Rev. D 39, 683 (1989).

[3] V. A. Kostelecký, R. Potting, Nucl. Phys. B 359, 545 (1991).

[4] B. Altschul, V. A. Kostelecký, Phys. Lett. B 628, 106 (2005).

[5] R. Gambini, J. Pullin, Phys. Rev. D 59, 124021 (1999). 
[6] J. Alfaro, H. A. Morales-Técotl, L. F. Urrutia, Phys. Rev. D 65, 103509 (2002).

[7] I. Mocioiu, M. Pospelov, R. Roiban, Phys. Lett. B 489, 390 (2000).

[8] S. M. Carroll, J. A. Harvey, V. A. Kostelecký, C. D. Lane, T. Okamoto, Phys. Rev. Lett. 87, 141601 (2001).

[9] V. A. Kostelecký, R. Lehnert, M. J. Perry, Phys. Rev. D 68, 123511 (2003).

[10] A. Ferrero, B. Altschul, Phys. Rev. D 80, 125010 (2009).

[11] F. R. Klinkhamer, C. Rupp, Phys. Rev. D 70, 045020 (2004).

[12] D. Colladay, V. A. Kostelecký, Phys. Rev. D 55, 6760 (1997).

[13] D. Colladay, V. A. Kostelecký, Phys. Rev. D 58, 116002 (1998).

[14] O. W. Greenberg, Phys. Rev. Lett. 89, 231602 (2002).

[15] R. Bluhm, V. A. Kostelecký, N. Russell, Phys. Rev. Lett. 79, 1432 (1997).

[16] G. Gabrielse, A. Khabbaz, D. S. Hall, C. Heimann, H. Kalinowsky, W. Jhe, Phys. Rev. Lett. 82, 3198 (1999).

[17] H. Dehmelt, R. Mittleman, R. S. Van Dyck, Jr., P. Schwinberg, Phys. Rev. Lett. 83, 4694 (1999).

[18] R. Bluhm, V. A. Kostelecký, N. Russell, Phys. Rev. Lett. 82, 2254 (1999).

[19] D. F. Phillips, M. A. Humphrey, E. M. Mattison, R. E. Stoner, R. F. C. Vessot, R. L. Walsworth , Phys. Rev. D 63, 111101(R) (2001).

[20] R. Bluhm, V. A. Kostelecký, C. D. Lane, Phys. Rev. Lett. 84, 1098 (2000).

[21] V. W. Hughes, et al., Phys. Rev. Lett. 87, 111804 (2001).

[22] B. R. Heckel, E. G. Adelberger, C. E. Cramer, T. S. Cook, S. Schlamminger, U. Schmidt, Phys. Rev. D 78, 092006 (2008).

[23] C. J. Berglund, L. R. Hunter, D. Krause, Jr., E. O. Prigge, M. S. Ronfeldt, S. K. Lamoreaux, Phys. Rev. Lett. 75, 1879 (1995).

[24] V. A. Kostelecký, C. D. Lane, Phys. Rev. D 60, 116010 (1999).

[25] D. Bear, R. E. Stoner, R. L. Walsworth, V. A. Kostelecký, C. D. Lane, Phys. Rev. Lett. 85, 5038 (2000).

[26] P. Wolf, F. Chapelet, S. Bize, A. Clairon, Phys. Rev. Lett. 96, 060801 (2006). 
[27] H. Müller, et al., Phys. Rev. Lett. 99, 050401 (2007).

[28] S. Herrmann, A. Senger, K. Möhle, E. V. Kovalchuk, A. Peters, in CPT and Lorentz Symmetry IV, edited by V. A. Kostelecký (World Scientific, Singapore, 2008), p. 9.

[29] S. Herrmann, et al., Phys. Rev. D 80, 105011 (2009).

[30] Ch. Eisele, A. Yu. Nevsky, S. Schiller, Phys. Rev. Lett. 103, 090401 (2009).

[31] H. Müller, Phys. Rev. D 71, 045004 (2005).

[32] G. Saathoff, S. Karpuk, U. Eisenbarth, G. Huber, S. Krohn, R. Muñoz Horta, S. Reinhardt, D. Schwalm, A. Wolf, G. Gwinner, Phys. Rev. Lett. 91, 190403 (2003).

[33] C. D. Lane, Phys. Rev. D 72, 016005 (2005).

[34] V. A. Kostelecký, Phys. Rev. Lett. 80, 1818 (1998).

[35] V. A. Kostelecký, Phys. Rev. D 61, 016002 (1999).

[36] Y. B. Hsiung, Nucl. Phys. Proc. Suppl. 86, 312 (2000).

[37] K. Abe et al. (Belle Collaboration), Phys. Rev. Lett. 86, 3228 (2001).

[38] J. M. Link et al., Phys. Lett. B 556, 7 (2003).

[39] B. Aubert et al. (BABAR Collaboration), Phys. Rev. Lett. 96, 251802 (2006).

[40] S. M. Carroll, G. B. Field, Phys. Rev. Lett. 79, 2394 (1997).

[41] V. A. Kostelecký, M. Mewes, Phys. Rev. Lett. 87, 251304 (2001).

[42] V. A. Kostelecký, M. Mewes, Phys. Rev. Lett. 97, 140401 (2006).

[43] V. A. Kostelecký, M. Mewes, Phys. Rev. Lett. 99, 011601 (2007).

[44] F. W. Stecker, S. L. Glashow, Astropart. Phys. 16, 97 (2001).

[45] T. Jacobson, S. Liberati, D. Mattingly, Nature 424, 1019 (2003).

[46] B. Altschul, Phys. Rev. Lett. 96, 201101 (2006).

[47] B. Altschul, Phys. Rev. D 74, 083003 (2006).

[48] F. R. Klinkhamer, M. Risse, Phys. Rev. D 77, 016002 (2008); addendum Phys. Rev. D 77, 117901 (2008).

[49] J. B. R. Battat, J. F. Chandler, C. W. Stubbs, Phys. Rev. Lett. 99, 241103 (2007). 
[50] H. Müller, S. W. Chiow, S. Herrmann, S. Chu, K.-Y. Chung, Phys. Rev. Lett. 100, 031101 (2008).

[51] V. A. Kostelecký, N. Russell, arXiv:0801.0287v6.

[52] V. A. Kostelecký, M. Mewes, Phys. Rev. D 69, 016005 (2004).

[53] P. Adamson, et al. (MINOS Collaboration), Phys. Rev. Lett. 101, 151601 (2008).

[54] P. Adamson, et al. (MINOS Collaboration), Phys. Rev. Lett. 105, 151601 (2010).

[55] P. Adamson, et al. (MINOS Collaboration), Phys. Rev. D 85, 031101(R) (2012).

[56] B. Rebel, S. Mufson, arXiv:1301.4684.

[57] L. B. Auerbach, et al. (LSND Collaboration), Phys. Rev. D 72, 076004 (2005).

[58] R. Abbasi, et al. (IceCube Collaboration), Phys. Rev. D 82, 112003 (2010).

[59] Y. Abe, et al. (Double Chooz Collaboration), Phys. Rev. D 86, 112009 (2012).

[60] A. A. Aguilar-Arevalo, et al. (MiniBooNE Collaboration), Phys. Lett. B 718 (2013).

[61] M. J. Longo, Phys. Rev. D 36, 3276 (1987).

[62] L. Stodolsky, Phys. Lett. B 201, 353 (1988).

[63] P. Adamson, et al. (MINOS Collaboration), Phys. Rev. D 76, 072005 (2007).

[64] T. Adam, et al., JHEP 1210, 093 (2012).

[65] B. Altschul, Phys. Rev. D 84, 091902(R) (2011).

[66] R. Bluhm, V. A. Kostelecký, C. D. Lane, N. Russell, Phys. Rev. D 68, 125008 (2003).

[67] R. Lehnert, R. Potting, Phys. Rev. Lett. 93, 110402 (2004).

[68] B. Altschul, Phys. Rev. Lett. 98, 041603 (2007).

[69] X.-J. Bi, P.-F. Yin, Z.-H. Yu, Q. Yuan, Phys. Rev. Lett. 107, 241802, (2011).

[70] L. Gonzalez-Mestres, arXiv:1109.6630.

[71] V. A. Kostelecký, M. Mewes, Phys. Rev. D 70, 076002 (2004).

[72] V. A. Kostelecký, M. Mewes, Phys. Rev. D 85, 096005 (2012).

[73] B. Altschul, Astropart. Phys. 28, 380 (2007). 